\title{
On-demand Take of Dapoxetine for the Treatment of Lifelong Premature Ejaculation: Prospective, Randomized, Placebo Controlled Study
}

\author{
Hong-yan LI ${ }^{1}$, Yuan-yuan XING ${ }^{2}$ and Hai LI ${ }^{3,{ }^{*}}$ \\ ${ }^{1}$ Department of Urology, China-Japan Union Hospital of Jilin University, Changchun \\ 130033, Jilin, China \\ Tel: +86-13756069386 Email: Lihongyan006@163.com \\ ${ }^{2}$ Department of Dialysis centers, China-Japan Union Hospital of Jilin University, \\ Changchun 130033, Jilin, China \\ ${ }^{3}$ Department of Urology, China-Japan Union Hospital of Jilin University, Changchun \\ 130033, Jilin, China
}

Tel: +86-13604321785 Fax: +86-431-89876971. Email: 624177683@qq.com

${ }^{*}$ Corresponding author

Keywords: Dapoxetine, Treatment, Lifelong premature ejaculation.

\begin{abstract}
The objective of this study is to assess the safety and efficacy of on-demand use of dapoxetine in treating lifelong premature ejaculation. This is a prospective, single center, single blind, placebo controlled study. During the period of months between january 2015 and july 2015,60 patients diagnosed with lifelong premature ejaculation(LPE) in outpatient department of China and Japan Union Hospital Of Jilin University, were randomized to receive different sequences of the two medications equally: placebo(group A), 30mg or $60 \mathrm{mg}$ of dapoxetine(group B) according to the efficacy at 8th week.Every patient received each medication for 12 weeks.Intravaginal ejaculatory latency time(IELT), premature ejaculation diagnostic tools(PEDT) score, sexual satisfaction score of patients and sexual partners were recorded during the study period. Successful of LPE is defined whether IELT exceed 120s. Meanwhile we observed the occurrence of adverse events in two groups.55 patients enrolled in our study completed the study over 12 weeks, 27 cases receive placebo and 28 cases receive $30 \mathrm{mg}$ or $60 \mathrm{mg}$ dapoxetine. Patients in group B had a significantly longer mean IELT than group $\mathrm{A}(120.4 \mathrm{~s}$ vs $42.1 \mathrm{~s}, \mathrm{P}<0.01)$. The average PEDT score in group $\mathrm{B}$ was different with group $\mathrm{A}(9.9$ vs $13.7, \mathrm{P}<0.01)$. Both of patients and sexual partners in group $\mathrm{B}$ felt more satisfied with their sexual life, which were better than group $\mathrm{A}(3.1$ vs 1.8,2.8 vs 1.9, respectively, $\mathrm{P}<0.01)$. 3 cases appeared dizziness and 1 case appeared nausea. There was no serious adverse event in any subjects. On-demand use of dapoxetine may be considered a safe and effective way in treating lifelong premature ejaculation. The long-term benefits need further study.

Premature ejaculation(PE) is the most common type of male sexual dysfunction disease,impacting $35 \%$ of global male, and the prevalence in china is between $35 \%$ and $50 \%[1]$.The data may have deviation due to the definition of premature ejaculation, clinic rate and so on. PE divided into lifelong premature ejaculation(LPE) and acquired premature ejaculation(APE) by early scholars. Recently, studies put forward variable PE (VPE) and subjective PE (SPE) after the two types above [2].According to the diagnostic criteria of PE published by international society of sexual medicine(ISSM), LPE characterized by:
\end{abstract}


ejaculation that always or nearly always occurs prior to or within about 1 minute of vaginal penetration from the first sexual experience; the inability to delay ejaculation on all or nearly all vaginal penetrations; and negative personal consequences, such as distress, bother, frustration, and/or the avoidance of sexual intimacy[3]. PE may affect many aspects of patient's life , including: self-confidence, interpersonal relationship, sexual satisfaction of both partners[4-5].What's more,the effects above worse the degree of PE in return. Several treatments have been used in clinical practice, mainly including: psychological therapy, behavior therapy, drug therapy, surgical treatment. Dapoxetine is a kind of selective serotonin reuptake inhibitors (SSRI), which take orally 1-3h prior to sexual life and can be absorbed and eliminated rapidly. Studies aim to treat LPE by on-demand take of dapoxetine were less well established. Moreover, most of them don't set control group especially placebo control group. In order to assess the value of on-demand take of dapoxetine in LPE, we conducted a prospective, single blind, placebo control study, studying the efficacy and safety.

\section{Subjects and Methods}

We totally selected 60 patient diagnosed with lifelong premature ejaculation in outpatient department of China and Japan Union Hospital Of Jilin University,from january 2015 to july 2015.All men were 20-50 years old;heterosexual;sexually active;stable sexual relationship for at least 6 months; fit the definition of LPE declared by ISSM above.Patients with erectile dysfunction; with psychiatric or systemic diseases such as diabetes mellitus; with malformation, trauma and infection of urinary reproductive system;with hypertension;with alcohol or substance abuse;or who used any drugs may cause or treat PE within 3 months were excluded.

The patient were randomly distributed into two groups(30 each).Group A patients were took placebo orally $1-3 \mathrm{~h}$ prior to sexual contact and stick to water more than $250 \mathrm{ml}$ at the same time. Patient in group B took dapoxetine $30 \mathrm{mg}$ orally in exactly the same way as group A.Patients in group B were assessed after 8weeks,IELT and adverse events were recorded.Patients in group B were distribute into three subgroup according to IELT and adverse events:30mg dapoxetine(IELT>120s, with mild adverse events or without adverse evens);60mg dapoxetine(IELT $<120 \mathrm{~s}$, with mild adverse events or without adverse events);stop drug(serious adverse events that patients can't tolerate).

All the patient were followed up after 12 weeks, recording their IELT, PEDT score and sexual satisfaction score(SSS) of both patients and partners, while observing the occurrence of adverse events.Sexual satisfaction was divided into extremely dissatisfied, dissatisfied, general, satisfied, extremely satisfied, corresponding to 1 to 5 points in turn. The data were

analyzed by $\mathrm{t}$ test using SPSS 13.0, measurement data show as $\bar{X} \pm S, \mathrm{P}<0.05$ was considered significance.

\section{Result}

55 patients enrolled in our study completed the study, 27 cases receive placebo and 28 cases receive $30 \mathrm{mg}$ or $60 \mathrm{mg}$ dapoxetine. The age of the patients varied from 23 to 43 years(mean $29.2 \pm 5.3,30.1 \pm 5.1$ years in group $\mathrm{A}, \mathrm{B}$, respectively). The age and body mass index between two group had no statistically difference $(\mathrm{P}>0.05)$, the data acquired in the end were comparable. 
At the end of 12 weeks, 27 patients in group A and 28 patients in group B were assessed.16 cases in group B took dapoxetine 30mg and the other 12 cases took dapoxetine $60 \mathrm{mg}$. There were no significant difference in the mean IELT, PEDT score, SSS of both patients and partners between the two groups posttreatment $(\mathrm{P}>0.05$, in table 1$)$, the data after treating were comparable.

Comparing the average IELT in different groups showed that dapoxetine-treat patient had a significantly longer IELT than group $\mathrm{A}(120.4 \mathrm{vs} 42.1 \mathrm{~s}, \mathrm{P}<0.01)$. Moreover, PEDT score in group B were better than group $\mathrm{A}(9.9$ vs $13.7, \mathrm{P}<0.01)$. Both of patients and sexual partners felt more satisfied with sexual life in group $B$, which were better than group $\mathrm{A}(3.1 \mathrm{vs} 1.8,2.8$ vs 1.9 , respectively, $\mathrm{P}<0.01$ ). The details can be seen in table2. Additionally, mean IELT, PEDT score and SSS of both patinets and partners in group were satisfactory after the treatment $(\mathrm{P}<0.01$,in table 3$) .18$ cases $(64 \%)$ in group $\mathrm{B}$ were effective in the end according to the effective indicators above.

Adverse events were reported by 4 men in group $\mathrm{B}(14.3 \%), 3$ cases appeared dizziness $(10.7 \%)$ and 1 cases appeared nausea (3.6\%), 1 of them appeared dizziness was observed when he increased the dosage of dapoxetine to $60 \mathrm{mg}$. These adverse events occurred in 1 week and disappeared within 2-3 weeks, there were no serious adverse events reported.

Table 1: intravaginal ejaculatory latency time(IELT), premature ejaculation diagnostic tools(PEDT) score, sexual satisfaction score(SSS) of patients and sexual partners before treat

\begin{tabular}{ccc}
\hline & Group A $(\mathrm{n}=27)$ & Group B $(\mathrm{n}=28)$ \\
\hline ILET & $41.3 \pm 11.3 \mathrm{~s}$ & $42.1 \pm 11.7 \mathrm{~s}$ \\
\hline PEDT score & $14.3 \pm 5.6$ & $14.2 \pm 4.2$ \\
\hline SSS of patients & $1.7 \pm 0.5$ & $1.6 \pm 0.6$ \\
\hline SSS of partners & $1.7 \pm 0.5$ & $1.7 \pm 0.6$ \\
\hline
\end{tabular}

Group A: subject with placebo;

Group B: subject with dapoxetine 30mg or 60mg on-demand;

Table2: intravaginal ejaculatory latency time(IELT), premature ejaculation diagnostic tools(PEDT) score,sexual satisfaction score(SSS) of patients and sexual partners after treat

\begin{tabular}{ccc}
\hline & Group A $(\mathrm{n}=27)$ & Group B $(\mathrm{n}=28)$ \\
\hline ILET & $52.9 \pm 16.2 \mathrm{~s}$ & $120.4 \pm 39.6^{*}$ \\
\hline PEDT score & $13.7 \pm 2.5$ & $9.9 \pm 2.4^{\star}$ \\
\hline SSS of patients & $1.8 \pm 0.7$ & $3.1 \pm 1.0^{*}$ \\
\hline SSS of partners & $1.9 \pm 0.7$ & $2.8 \pm 0.9^{\star}$ \\
\hline
\end{tabular}

Group A: subject with placebo;

Group B: subject with dapoxetine $30 \mathrm{mg}$ or $60 \mathrm{mg}$ on-demand;

${ }^{*} \mathrm{P}<0.01$

Table3: intravaginal ejaculatory latency time(IELT), premature ejaculation diagnostic tools(PEDT) score, sexual satisfaction score(SSS) of patients and sexual partners of group B during the study period

\begin{tabular}{lll}
\hline & Before Treat & After Treat \\
\hline ILET & $42.1 \pm 11.7 \mathrm{~s}$ & $120.4 \pm 39.6^{\star}$ \\
\hline PEDT score & $14.2 \pm 4.2$ & $9.9 \pm 2.4^{\star}$ \\
\hline SSS of patients & $1.6 \pm 0.6$ & $3.1 \pm 1.0^{\star}$ \\
\hline SSS of partners & $1.7 \pm 0.6$ & $2.8 \pm 0.9^{\star}$ \\
\hline
\end{tabular}

$* \mathrm{P}<0.01$ 


\section{Discussion}

PE is a common type of male sexual dysfunction disease.It can be a cause of personal distress which may also affect the relationship with their partners. While many patients puzzled by PE don't seek any medical measures, if they do, psychological therapy, behavior therapy, drug therapy and surgical therapy will be used in the treatment.Previously, PE was considered to be a disease related with psychological problems[6]. Psychotherapy involve education both patients and their partners, which may improve their sexual skills and increase self-confidence and reduce anxiety during sex[7].However, the majority of studies aim to treat PE by psychotherapy didn't meet the criteria for high level evidence based studies[8].The effectiveness of behavior therapy reported was different, with poor compliance of the patient.Although their utility are limited today, they are used to treat PE combined with drugs[9]. Surgical therapy such as ablation and modulation of dorsal penile nerve[10] is an innovative modality need further study and may cause irreversible damage which limit its clinical application.Circumcision seems to have some role in treating PE which can extend the ejaculation time[11],but it could not be interpreted as a justification for circumcision in men with PE even there were no adversely affect ejaculatory function[12]. Although drug therapy is widely used for the treatment of PE in clinic, few studies devote to on-demand use of dapoxetine to treat LPE.

The etiological factors of PE are various, involves psychology, environment, endocrine and neural physiological. The neural control of PE are concerned with a variety of neurotransmitters, studying widely of serotonin, considering $1 \mathrm{a}, 1 \mathrm{~b}$ and $2 \mathrm{c}$ receptors are associated with PE[13].For example, Ahlenius et al.[14] consider that stimulating 5-HT2C receptors delayed ejaculation, and reported the stimulus of 5-HT1A receptors in rats resulted in rapid ejaculation.However,the mechanism how the short-acting SSRIs treat PE is still speculative, scholars point out that they can't or only rarely increase 5-HT level in synaptic cleft[15].It is possible that short-acting SSRIs have other effects still unknown which play a role in treating PE. Dapoxetine is a selective serotonin reuptake inhibitor which can be absorbed quickly and reach the peak plasma concentration approximately $1 \mathrm{~h}$ after using, the half-life is $1.4 \mathrm{~h}$, the plasma concentration decrease to $5 \%$ of the peak after $24 \mathrm{~h}[16]$. Dapoxetine can be metabolized fast which enable it treat PE on-demand. This study found that on-demand use of dapoxetine can significantly extend IELT, perfect the PEDT score, improve the SSS of both patients and partners, comparing to the placebo control group $(\mathrm{P}<0.01)$. The average IELT in group B was about 2.9 folds compared to the initial data, which consistent with previous studie[17]. 18 cases(64\%) treated in group B were effective, the others discontent the outcome and the further treatment need further research.Ozbek $\mathrm{E}$ et al observed that the $\operatorname{short}(\mathrm{S})$ allele of serotonin transporter gene-linked polymorphic region(5-HTTLPR) was more frequent in responders than in nonresponders and concluded that PE patients with SS genotype were easier to have a good outcome when treated by SSRIs[18].HTR1A gene polymorphism is assocaited with IELT in LPE patients, the IELT of patients with CC genotype were shorter than the patients with GG or CG genotypes for the $\mathrm{C}(1019) \mathrm{G}$ polymorphism[19].The patients traeated invalid may associated with gene polymorphism. Several studies show that dapoxetine plus mirodenafil can be more effective than dapoxetine alone[20], phosphodiesterase type 5 inhibitors(PDE-5I) may have a potential 
role in the treatment of PE without ED which need further study.Moreover, Kim YH and his colleagues pointed out that there was no clinically significant pharmacokinetic interactions between dapoxetine and udenafil which is a sort of PDE-5Is[21].SSRIs plus PDE-5I may be a further direction for the treatment of PE even of LPE and can be well tolerated.

The most common adverse events of dapoxetine are headache, dizziness, nausea, dry mouth, diarrhea and vomiting. We observed 28 cases, 3 cases with dizziness $(10.7 \%)$ and 1 case with nausea(3.6\%), 1 of the 3 cases appeared after using dapoxetine 60mg. These adverse events occurred in 1 week and disappeared within 2-3 weeks. We didn't see anyone suffer from dry mouth which may be associated with take much water when have drugs. The incidence of adverse events of dapoxetine is relate to the dose,on-demand use of dapoxetine $30 \mathrm{mg}$ and $60 \mathrm{mg}$ for patients with LPE can be tolerated.Mondaini $\mathrm{N}$ et al showed $20 \%$ of patients with LPE seek for treatment refused pharmacotherapy by dapoxetine, and nearly $90 \%$ of patients started therapy discontinued after 1 year[22].

SSRIs are widely used in the treatment of PE, dapoxetine can be absorbed and metabolized quickly compare to the traditional SSRIs. On-demand use of dapoxetine to treat PE is effective which also can reduce the dosage and decrease the incidence of adverse events. Our study show that it's effective to treat LPE by on-demand use of dapoxetine, and the adverse events are mild and can be tolerated. We also suggest that $60 \mathrm{mg}$ dapoxetine should be given directly in cases of severe LPE with high expectation. All in all, on-demand use of dapoxetine for the treatment of LPE is safe and effective. Although short-term results are promising, the long-term benefits are unproven.

\section{References}

[1]Montors iF.Prevalence of premature ejaculation: a global and regional perspective . J Sex Med, 2005,5:96-102.

[2]Waldinger M. Premature ejaculation: Different pathophysiologies and etiologies determine its treatment. J Sex Marital Ther, 2008,34:1-13.

[3]Althof SE1, McMahon CG, Waldinger MD, et al. An update of the international society of sexual medicine's guidelines for the diagnosis and treatment of premature ejaculation (PE). J Sex Med,2014,11(6):1392-1422.

[4]Giuliano F, Patrick DL, Porst H, et al.Premature ejaculation: Results from a five-country European observational study. Eur Urol,2008,53:1048-1057

[5]Patrick DL, Althof SE, Pryor JL, et al. Premature ejaculation: An observational study of men and their partners. J Sex Med,2005,2:358-367.

[6]Jannini EA, Simonelli C, Lenzi A. Sexological approach to ejaculatory dysfunction. Int J Androl, 2002,25(6):317-323.

[7]Althof SE1.Psychological treatment strategies for rapid ejaculation: rationale, practical aspects, and outcome.World J Urol,2005,23(2):89-92.

[8]Althof S. Psychological approaches to the treatment of rapid ejaculation. J Mens Health Gend ,2006,3:180-186. 
[9]Cooper K, Martyn-St James M, Kaltenthaler E, et al. Behavioral Therapies for Management of Premature Ejaculation: A Systematic Review.Sexual Medicine. 2015,3 (3):174-188.

[10]Basal S, Goktas S, Ergin A,et al. A novel treatment modality in patients with premature ejaculation resistant to conventional methods: the neuromodulation of dorsal penile nerves by pulsed radiofrequency. J Androl,2010,31:126-130.

[11]Alp BF, Uguz S, Malkoc E, et al.Does circumcision have a relationship with ejaculation time? Premature ejaculation evaluated using new diagnostic tools.Int $\mathrm{J}$ Impot Res,2014,26(24):121 - 123.

[12]Morris BJ, Krieger JN.Does male circumcision affect sexual function, sensitivity,or satisfaction?--a systematic review.J Sex Med, 2013,10 (11): 2644- 2657.

[13]Patel K, Hellstrom WJ. Central regulation of ejaculation and the therapeutic role of serotonergic agents in premature ejaculation. Curr Opin Investig Drugs, 2009,10(7):681-690.

[14]Ahlenius S, Larsson K, Svensson L et al.Effects of a new type of 5-HT receptor agonist on male rat sexual behavior. Pharmacol Biochem Behav,1981,15: 785-792.

[15]Waldinger MD,Schweitzer DH,Olivier B.On-demand SSRI treatment of premature ejaculation:pharmacodynamic limitations for relevant ejaculation delay and consequent solutions.Journal of SEXUAL MEDICINE,2005,2(1):121-123.

[16]Modi NB, Dresser MJ,Simon M,et al.Single-and multiple-dose pharmacokinetics of dapoxetine hydrochloride, a novel agent for the treatment of premature ejaculation.J Clin Pharmacol,2006,46(3):301-309.

[17]McMahon CG, Althof SE, Kaufman JM, et al. Efficacy and safety of dapoxetine for the treatment of premature ejaculation: Integrated analysis of results from five phase 3 trials. J Sex Med,2011,8:524-539.

[18]Ozbek E, Otunctemur A, Simsek A, et al.Genetic polymorphism in the serotonin transporter gene-linked polymorphic region and response to serotonin reuptake inhibitors in patients with premature ejaculation.Clinics (Sao Paulo). 2014,69(11):710-713.

[19]Janssen PK1, van Schaik R2, Zwinderman AH.The 5-HT 1 A receptor C(1019)G polymorphism influences the intravaginal ejaculation latency time in Dutch Caucasian men with lifelong premature ejaculation.Pharmacol Biochem Behav, 2014, 121:184-188.

[20]Lee WK1,Lee SH,Cho ST, et al.Comparison between on-demand dosing of dapoxetine alone and dapoxetine plus mirodenafil in patients with lifelong premature ejaculation:prospective,randomized,double-blind, placebo-controlled, multicenter study . J Sex Med,2013,10(11):2832-2841.

[21]Kim YH, Choi HY, Lee SH,et al.Pharmacokinetic interaction between udenafil and dapoxetine: a randomized, open-labeled crossover study in healthy male volunteers.Drug Des Devel Ther, 2015,23(9):1209-1216

[22]Mondaini N, Fusco F, Cai T, et al.Dapoxetine treatment in patients with lifelong premature ejaculation: the reasons of a "Waterloo".Urology,2013,82(3):620-624. 